\title{
LAS INSCRIPCIONES FENICIAS DEL TELL DE DOÑA BLANCA (IV) *
}

JESÚS-LUIS CUNCHILLOS

CSIC. Madrid

TDB 82003(?)

$1^{9} \quad$ Número de inventario. No consta.

$2^{\circ}$ Contexto arqueológico. Región suroriental de la ciudad.

$3^{\circ}$ Descripción del objeto o soporte de la escritura. Fragmento de cerámica de pasta marrón claro, beige, con engobe rojo en el interior. Fragmento de un plato fenicio del siglo VII. Presenta fracturas por los cuatro costados o lados. Mide $32 \mathrm{~mm} . \times 48 \mathrm{~mm}$. $\times 25 \mathrm{~mm}$.

$4^{2}$ Ésta es su fotografla con testigo centimetrado.

* A este artículo preceden los siguientes «Las inscripciones fenicias del Tell de Doña Blanca (I). Primera aproximación", enviado para su publicación en las actas del Simposio Internacional de Cartagena (17-19 de noviembre 1990). En el citado artículo, primero de la serie, damos las indicaciones de método adoptadas; "Las inscripciones fenicias del Tell de Doña Blanca (II)" aparecido en Sefarad 51 (1991) 13-22; "Las inscripciones fenicias del Tell de Doña Blanca (III)" en AuOr 8 (1990) 175-181. Aprovecho la ocasión para dar las gracias al Prof. Diego Ruiz Mata, Director de la excavación de TDB, y a sus ayudantes los arqueólogos Carmen J. Pérez y Francisco Barrionuevo, sin cuya cooperación no hubiera sido posible dar la información arqueológica que se contiene en éste y en los precedentes artículos. 


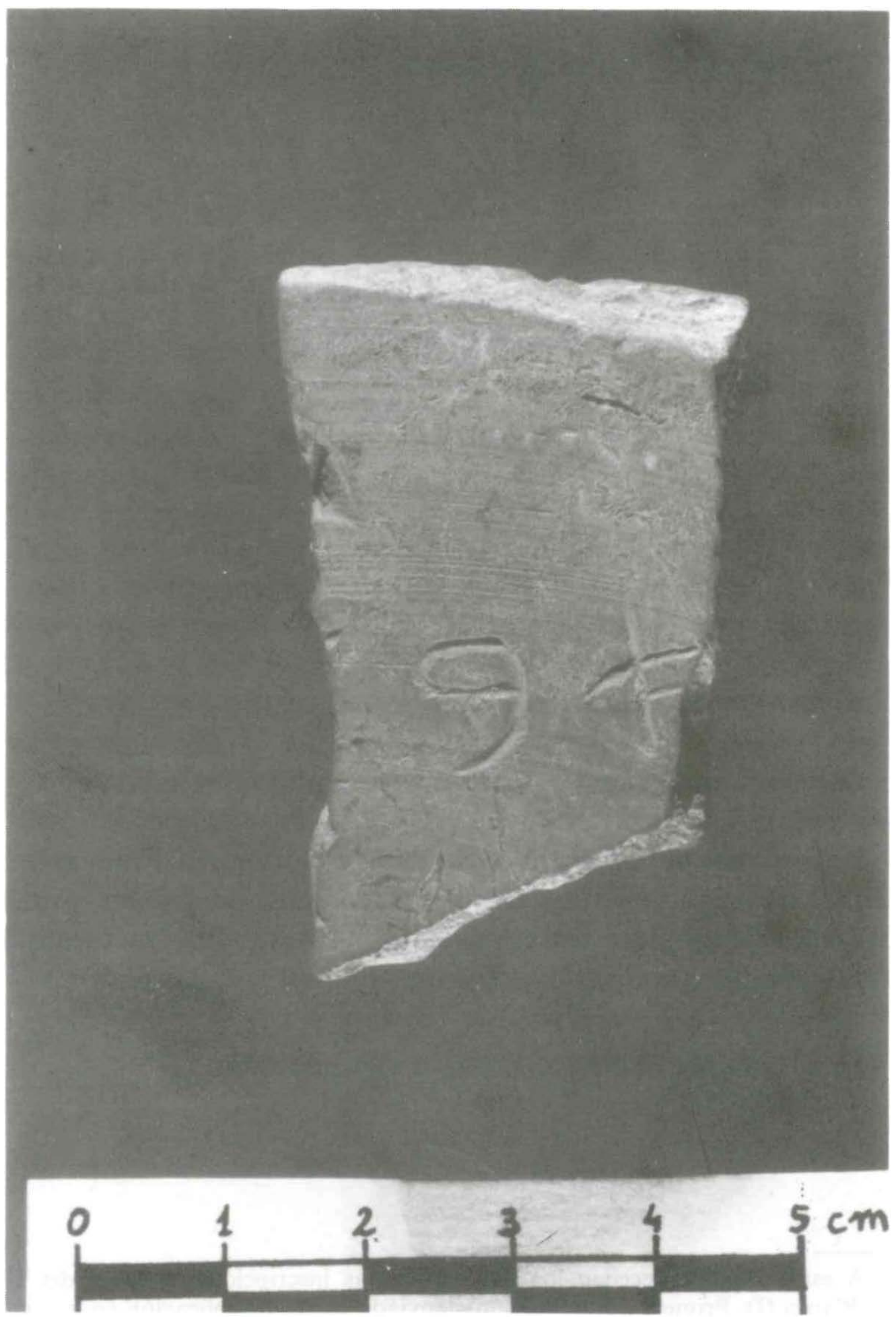

Fotografía de TDB 82003

(c) Consejo Superior de Investigaciones Científicas

Reconocimiento 4.0 Internacional (CC BY 4.0) 
$5^{0} \quad$ Y éste es el dibujo de la pieza.
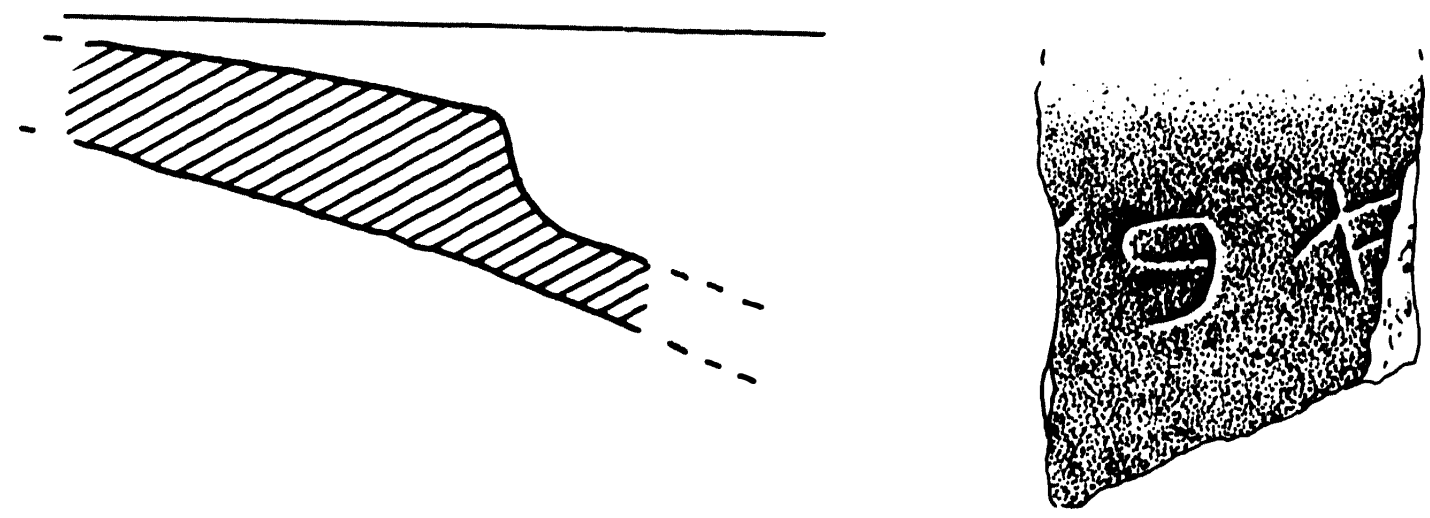

Dibujo de TDB 82003

69 La inscripción. Se encuentra en el exterior, parte convexa de la pieza. La incisión ha sido realizada a mano antes de la cocción con un punzón de punta redonda, de derecha a izquierda y de arriba abajo.

El primer signo es un' tipo F-R $n^{2} 15^{\prime}$. Inmediatamente antes, una de las fracturas nos impide saber si la palabra comenzaba por'. Al ` sigue una b bastante redonda. La separación entre ambos signos es de $6 \mathrm{~mm}$. Ahora bien, el espacio existente entre la b y la fractura situada a su izquierda es de $5 \mathrm{~mm}$., lo que no permite tampoco concluir si ab representa la inscripción en su totalidad. Una pequeña muesca a la izquierda de la $b$ y al borde de la fractura debe interpretarse como comienzo de un tercer signo. La inscripción hay que leerla:

\section{]'bxì}

${ }^{1}$ Para que el lector pueda hacerse una idea más precisa de la morfología de los grafemas, y mientras no dispongamos de tablas epigráficas de TDB, daremos referencias a la Schrifttafel de J. FrIEdRICH - W. ROLlig, Phonizisch-Punische Grammatik, (Analecta Orientalia 46) Roma 1970, al final del volumen. A partir de ahora nos referiremos siempre a esa tabla bajo la sigla F-R $n^{2}$. 
$7^{0}$ Interpretación. La fractura anterior y posterior a la zona legible impide dar una interpretación cierta. Pueden apuntarse algunas sugerencias. Se podría pensar en 'bst 'tazón' 2 , pero el fragmento no corresponde a una copa o tazón. Cabe la posibilidad de que la misma palabra 'bst signifique '[plato o recipiente] para comer' fundándose en el hebreo ${ }^{3}$.

Pero la intepretación más prudente verá en 'bxx[ el comienzo de un antropónimo, de los que existen varios atestiguados ${ }^{4}$. Entre ellos 'b', antropónimo fenicio atestiguado en RES $620^{5}$, un sello procedente de Beirut ${ }^{6}$, y en otras inscripciones orientales como un sello procedente de Jerusalén ', en un sello cananeo antiguo del valle de Ayalón ${ }^{8}$ y en España en la inscripción Hispania 16 proveniente de Toscanos ${ }^{9}$, cerca de Torre del Mar en la provincia de Málaga. La inscripción de Toscanos tiene como soporte un fragmento de plato con engobe rojo, perteneciente al estrato IV de principios del siglo VII según escribe Solá-Solé ${ }^{10}$. El tipo de cerámica es el mismo, pero la forma del 'alif es diferente e incluso la forma de la b, más redondeada en TDB.

$8^{\circ}$ Fecha. Entre el 650 a. C., según el tipo de cerámica y el 590 a. C. según la aparente paleografía.

TDB 87001

19 Número de inventario. TDB 87/Espigón 01.1/GK/1.

\footnotetext{
${ }^{2}$ Véase R. S. Tomback, A Comparative Semitic Lexicon of the Phoenician and Punic Languages, Missoula, Montana 1978, pág. 3.

3 Véase R. S. Tomback, ibidem, pág. 3.

${ }^{4}$ Véase F. L. BEnZ, Personal Names in the Phoenician and Punic Inscriptions, Roma 1972, págs. 54 y 55.

${ }^{5}$ Véase F. L. Benz, o.c., pág. 54.

${ }^{6}$ Véase también K. Galling, ZDPV 64 (1944) pág. 173, n 5.

7 Véase S. Moscatl, L'Epigrafia Ebraica Antica, Roma 1951, pág. 65, n 42.

${ }^{8}$ Véase R. Giveon, "Two New Hebrew Seals and Their Iconographic Background", $P E Q 93$ (1961) págs. 38-39.

9 Véase J. M. SolÁ-SolÉ, "Textos epigráficos de Toscanos", Madrider Mitteilungen 9 (1968) pág. 106 y Tafel 32c al final del volumen. Véase también W. Röllig, "Contribución de las inscripciones fenicio-púnicas al estudio de la protohistoria de España", AuOr 4 (1986) pág. 56.
}

${ }^{10}$ Ibidem. 
$2^{\mathrm{o}}$ Contexto arqueológico. En la parte suroriental del tell. Vertiente norte del espigón. En un estrato potente de basura que colmata el relleno de piedras de las casas fenicias ya caídas. Los arqueólogos fechan este estrato en la segunda mitad del siglo VIII a. C.

$3^{\text {o }}$ Descripción del objeto o soporte de la escritura. Fragmento de un borde de pátera o cuenco carenado del siglo VIII a. C. La vasija estaba barnizada con engobe rojo en su interior y hasta la carena en el exterior. Mide $76 \mathrm{~mm}$. × 39,5 mm. y un espesor de $7 \mathrm{~mm}$. Factura y decoración local, salvo análisis de pasta.

4ํ Ésta es la fotografía de la pieza con testigo centimetrado.

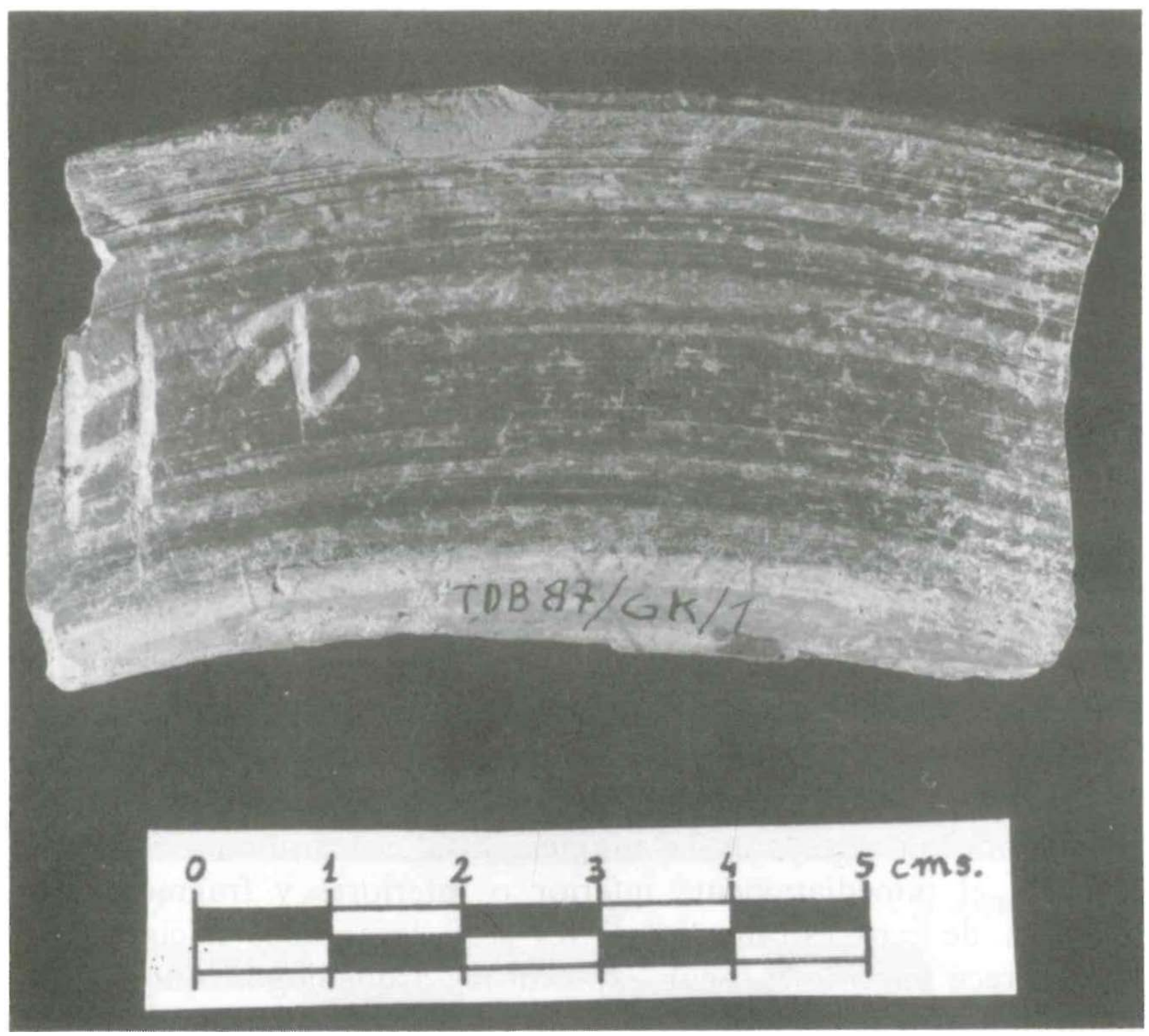

Fotografía de TDB 87001 
$5^{\varrho} \quad$ Éste es el dibujo de la pieza.
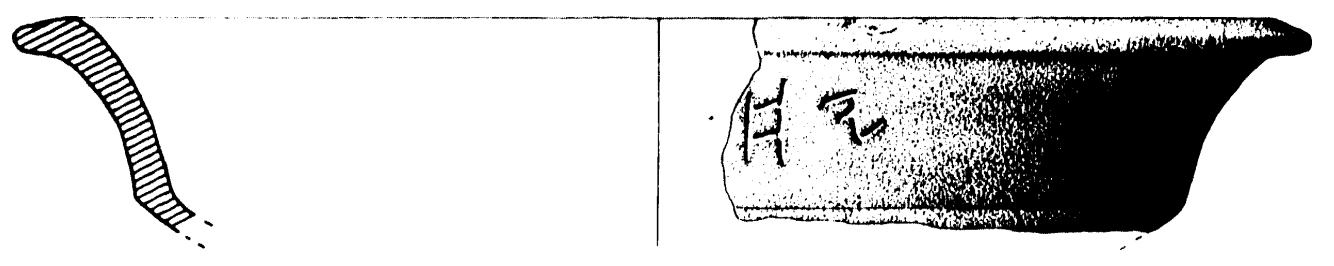

Dibujo de TDB 87001

69 La inscripción se encuentra en la parte exterior de la boca de la vasija. La inscripción es posterior a la cocción, posterior al barnizado o engobe porque el trazo, siendo amplio, no está barnizado o engobado en ningún momento. La sección del punzón es muy ancha, $2 \mathrm{~mm}$., y un tanto burda. Las letras no están bien alineadas entre sí. La inscripción comenzaba ciertamente con $\mathrm{y}$, corresponde al tipo F-R $n^{2} 156 n^{2} 16$. Sigue una $h$ indiscutible, pero de sólo dos trazos horizontales, lo que es relativamente raro, pero encontraremos otros casos en TDB. La h se parece en parte a F-R $n^{2} 15 / 3$ o al $n^{2}$ 33. La lectura de la inscripción es:

$\mathbf{y b}[$.

79 La interpretación. La lectura de la inscripción yhr puede hacer pensar en una forma verbal yqtl, perteneciente a un antropónimo teóforo del tipo yhmlk "Que Milk viva!»", entre los que están atestiguados: $y h w^{2}, y h w^{2} l n, y h w l n, y h w m l k, y h z b<l, y h l b<l, y h m l k$, $y h n b<l, y h r b<l 12$.

$8^{2}$ Fecha. Si el estrato es de la segunda mitad del vill a. C., dificilmente se le puede dar a la incripción una fecha posterior. Se puede concebir que ya en la antigüedad, al constituir un estrato se remueva el inmediatamente inferior o inferiores y fragmentos de cerámica de esos estratos inferiores sean levantados hacia arriba. Pero parece imposible, según explican los arqueólogos, que el fragmento pueda ir hacia abajo, hacia un estrato ya constituido.

$"$ Véase F. L. Benz, o. c., págs. 208-210.

12 Véanse referencias en F. L. BENZ, o.c., págs. 127-128. 
TDB 87002

1N Número de inventario TDB 87 Espigón 01.1/DP/3.

$2^{\circ}$ Contexto arqueológico. Fragmento encontrado en la parte suroriental del tell, en la vertiente norte del espigón. Estrato de la segunda mitad del siglo vill a. C. El mismo estrato en el que apareció TDB 87001.

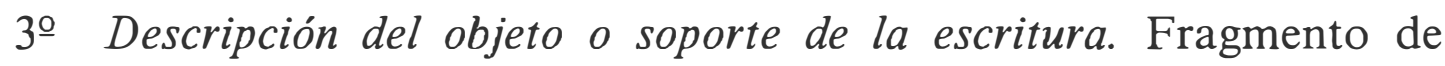
arcilla blanca amarillenta en el exterior y rojiza en el interior. Mide $44,5 \mathrm{~mm} . \times 38 \mathrm{~mm}$. Pertenece al hombro de un ánfora de tipo estrictamente oriental, tipo Sagona 2. Este tipo de ánforas se utilizaba para contener y transportar vino o aceite.

4o Ésta es la fotografía de la pieza con testigo centimetrado.

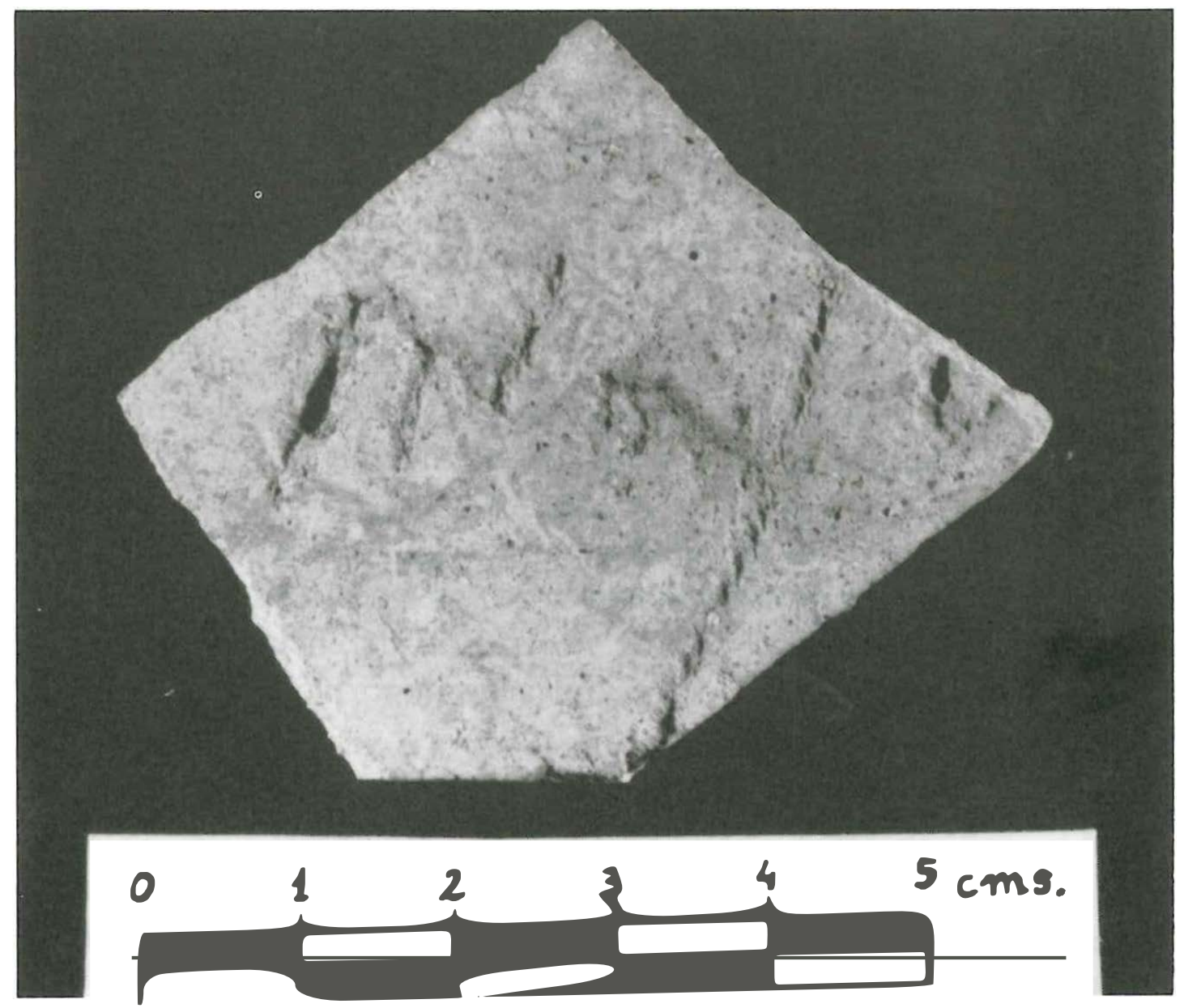

Fotografía de TDB 87002 
$5^{9} \quad \mathrm{Y}$ éste es el dibujo de la pieza.
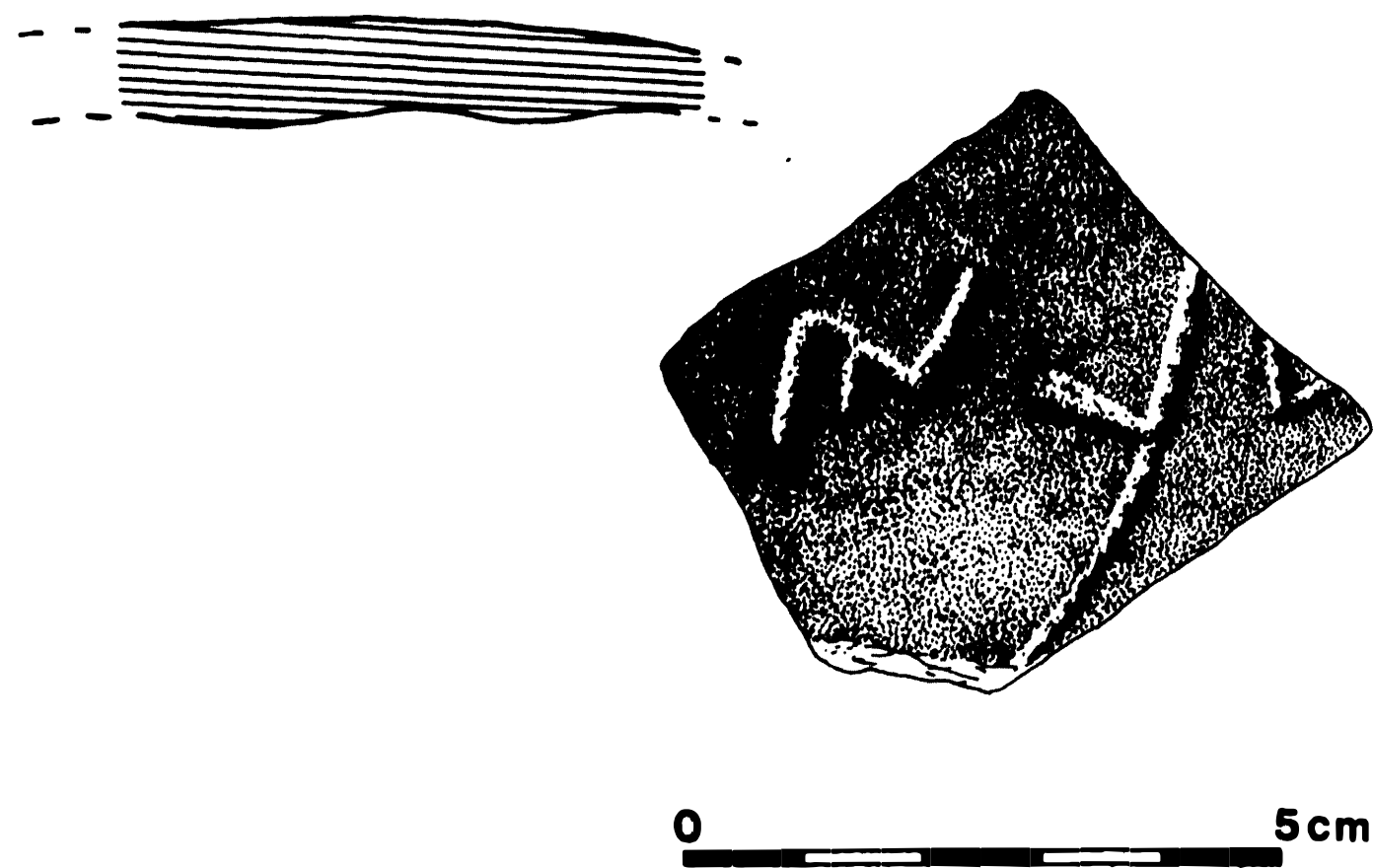

Dibujo de TDB 87002

69 La inscripción. La incisión ha sido hecha, cuando la arcilla estaba blanda, con un estilete ocasional o fortuito en el hombro del ánfora. La incisión es muy tosca. El trazo tiene hasta $3,9 \mathrm{~mm}$. de ancho.

A la derecha se lee la mitad de un c, sigue una $\mathbf{k}$ casi completa y se ve perfectamente una $y$. La letra y paleográficamente puede ser del tipo F-R $n^{2} 10$ (825 a. C.). Nótese la diferencia de inclinación del y en esta inscripción (de cerámica de importación) y la anterior TDB 87001 (cerámica indígena). Su lectura es:

cky

79 Interpretación. La inscripción reza cky, es decir, el topónimo Acre, bien atestiguado en la antiguedad ${ }^{13}$, llamado más tarde $\mathrm{S}$. Juan de Acre, en Líbano, en plena costa fenicia.

${ }^{13}$ Véase por ejemplo J. L. CUNChillos, "Correspondance", en Textes Ougaritiques II, (Littératures anciennes du Proche Orient, 14) pág. 357, nota 30. Doy las gracias a Corinne Bonnet que me ayudó en la lectura de esta inscripción. 
Siendo la cerámica de importación y la inscripción hecha cuando la pasta estaba blanda, hay que concluir que la inscripción se hizo en el mismo lugar de la producción. Si la inscripción no señala el lugar de producción, lo que es probable, al menos indica el origen del ánfora: Acre, en Fenicia. La fecha de su producción es un poco anterior a la segunda mitad del siglo VIII, es decir, por lo menos del 750 a. C.

La diferencia de inclinación del y en esta inscripción (de cerámica de importación) y la anterior TDB 87001 (cerámica indígena) parece indicar que, o bien TDB 87002 es anterior a TDB 87001 , o que hay variantes en la paleografía fenicia de Oriente y de Occidente en la segunda mitad del siglo VIII, o más exactamente entre la primera mitad del siglo VIII (TDB 87002) y la segunda mitad del mismo siglo (TDB 87001).

$8^{\circ} \quad$ Fecha. Anterior a la segunda mitad del siglo vili a. C. Probablemente primera mitad del siglo VIII a. C.

\section{RESUMEN}

El artículo propone la lectura e interpretación de tres inscripciones aparecidas en el Tell de Doña Blanca (Puerto de Santa María, Cádiz). Las dos primeras reproducen, probablemente, el comienzo de dos antropónimos. La tercera, anterior al 750 a. C., es un fragmento del hombro de un ánfora fenicia, Sagona 2, en la que aparece inscrito el topónimo Acre.

\section{SUMMARY}

The present article proposes the reading and the interpretation of three inscriptions that were discovered at the Tell de Doña Blanca (Puerto de Santa María, Cádiz). The first two ones probably reproduce the beginning of two anthroponyms, while the third one, dating back to a period prior to $750 \mathrm{BC}$, is a fragment of the shoulder of a Phoenician amphora (Sagona 2), showing the inscription of the toponym Acre. 